\title{
ARCHITECTING THE SMART GRID AS A HOLARCHY
}

\author{
Ebisa Negeri and Nico Baken \\ Delft University of Technology, Delft, The Netherlands
}

Keywords: Smart Grid, Architecture, Prosumer.

Abstract: The ever increasing concerns for energy security, energy efficiency, and sustainable energy is offering various challenges for the power grid. With increasing penetration of the distributed generations, the electricity power system is facing an era of prosumerization, whereby all stakeholders can autonomously produce, consume, import and/or export power. The classical power grid with top-down organization and control does not fit this dynamics, hence reorganizing the rather old architecture of the system is indispensable. In this paper, we propose a generic architecture of the smart grid that fits the new scenario based on the concepts of holons. The proposed architecture of the system is composed of autonomous prosumers that are organized bottom-up in a recursive manner involving various aggregation layers, forming a dynamically reconfigurable system. A corresponding control architecture that employs a holonic approach to simultaneously capture the autonomy of the prosumers, the recursion and the dynamic reconfiguration of the proposed system is also proposed. We extend our work by proposing a service oriented architecture (SOA) framework to support our control architecture.

\section{INTRODUCTION}

With the increasing need of enhancing the share of renewable energy, energy security and efficiency, distributed sources are increasingly penetrating into the electricity grid. Consequently, the lower end points of the grid are "taking power into their own hands" as they own distributed sources. For instance, households are evolving from passive consumers to active prosumers that can generate, store, import or export power. According to the European parliament, all new buildings to be built after 2019 will have to produce their own energy on site (European Parliament (2009)). The electric vehicles and the future fuel cell vehicles could become mobile power storage and generation elements of the grid. These trends imply that the power system is facing an era of prosumerization, whereby all stakeholders can autonomously produce, consume, import and/or export power on the grid. In this work, prosumer is a general term that refers to a system that autonomously manages its resources and is capable of bidirectionally exchanging power with its environment.

While prosumers autonomously manage their own resources, they can join other prosumers whose profile "complement" their own to exchange power with them. Further, a group of such prosumers might constitute a larger prosumer cluster to gain the power of the collective and bargain with the rest of the grid as a unit. Yet, a group of prosumer clusters can form a bigger prosumer cluster to benefit the power of the bigger collective. In this era of prosumerization, it seems that the future power system builds bottom-up. Thus, restructuring the rather old centralized top-down organized power system is crucial to accommodate the dynamics.

In this paper, we propose a generic architecture of the smart grid and its corresponding control architecture that can efficiently manage the era of prosumerization. Our proposed architecture employs holonic approach to organize the system bottom-up by aggregating the prosumers at different aggregation layers. The proposed architecture incorporates dynamic reconfiguration capability allowing the system to adapt to changes. We have also proposed a control architecture that well suits the envisioned system. Further, a service-oriented architecture (SOA) bases framework that models the control application of our proposed system is presented.

The rest of this paper is organized as follows. After we present the related background of the topic in Section 2, we present our proposed architecture of the system in Section 3. Our holonic control 
architecture and its SOA based model are present in Section 4. In Section 5, we describe the conditions required to realize the proposed architecture. Finally, we present the concluding remarks of the paper in Section 6 .

\section{BACKGROUND}

\subsection{Recent Concepts in Power System}

Recently, various new concepts have been proposed to manage the new trends in the power system. The Flexible, Reliable, and Intelligent Electrical eNergy Delivery System (FRIENDS) (Nara and Hasegawa, 1997) was proposed aiming to create a new framework for power system that realizes flexible reconfiguration of the system using switching operation, multiple menu service to allow customers to select the quality of power, and demand side management. The concept of Virtual Power Plants (VPP) was introduced as a cluster of distributed power sources which are collectively managed (Dielmann and van der Velden, 2003). The Microgrid concept (Hatziargyriou et al., 2006) was suggested as a low voltage (LV) distribution system comprising of distributed sources, controlled loads and storage systems that are coordinated to achieve a controllable operation either as an island or connected to the medium voltage (MV) grid.

Another concept is the autonomous network (AN), which is a part of the system but its behavior is more or less independent from the rest (Provoost et al., 2004). AN differs from the microgrid because AN is larger in size and complexity, as well as its aim is primarily optimizing its normal operation. In (van Overbeeke and Roberts, 2002), the active networks concept is presented where the network is subdivided into cells that are self managing, but not necessarily self supplying. This concept involves interconnection between the cells that provides more than one power flow paths, and allows rerouting to avoid congestion and to isolate faults. Further, system services are traded along the connections between the cells.

The smart grid concept represents the overall picture of the future power network that is supported by intelligent distributed devices and communication technologies. In (Tsoukalas and Gao, 2008), the smart grid is modeled as an energy internet comprising of local area grids (LAGs), that are demand-based autonomous entities consisting of a convenient mixture of different customers. The LAGs interact with the system using their intelligent agents. The Future Renewable Electric Energy Delivery and Management (FREEDM) (Huang et al., 2011) have been proposed as a power distribution system that interfaces with residential customers and industry customers having distributed renewable energy sources and distributed storage devices. The key technology features of the system are the plug-and-play interface, energy router, and open-standard based operating system. The proposed system relies on a flat and distributed management architecture.

A market based control concept, named PowerMatcher, is suggested for supply and demand matching in electricity networks with large penetration of distributed sources (Kok et al., 2005). In the PowerMatcher, each device uses its agent to buy or sell power in the electronic market that is implemented in a distributed manner using a tree structure. The device agents can also be clustered to form intermediated aggregator agents. In (Grijalva and Tariq, 2011), it is argued that each component of the power system could be considered as a prosumer, based on which they model the electricity power system as a flat system composed of prosumers of different scales. Correspondingly, they propose a totally flat control architecture of the system.

Although their scale, complexity and intelligence varies, all the above concepts and technologies have their own contributions in managing the distributed sources. One concept could be more applicable in some settings than the other, and vice versa. Moreover, some of the concepts could be combined since they complement each other. The smart grid could be an umbrella to appropriately combine the concepts, thereby achieve a heterogeneous and intelligent power system that delivers its expectations. For instance, the FRIENDS, VPP, microgrid, AN, and active networks could form subnetworks of the smart grid, while FREEDM provides the power delivery infrastructure supporting the sub-networks and PowerMatcher delivers the transaction services.

In this work, we endeavor to propose a generic architecture of the smart grid that tends to converge the various proposed concepts. Our proposed architecture appropriately accommodates the heterogeneous composition of the smart grid as well as provides flexibility, intelligence and autonomy at all level of the grid as required.

\subsection{Holons and Holarchy}

The word holon was coined by (Koestler, 1990), and 
it refers to a logical entity that is both a whole and a part. For example, a cell in your body is a holon because it is a distinct living entity that has a distinct cell wall defining its interface with the rest of the world. However, a cell is composed of smaller holons such as nucleus and chromosome that are also separate entities. Yet, a group of cell holons together form a higher aggregation layer tissue holon, a group of tissue holons form an organ holon, and so on.

A holon is basically autonomous, cooperative and recursive. Holons exhibit self-similar recursive structure and are organized in various aggregation layers to form a complete holarchy as shown in Figure 1. In a holarchy, each holon can function autonomously thereby enabling formation of a distributed system. While functioning autonomously, a holon can cooperate with other holons in the holarchy to achieve mutual goals. In a dynamic environment, holons can also be dynamically reorganized thereby making the holarchy more robust to changes adapting itself to its environment. Recently, there is a growing interest in the holonic approach in the development of various systems (e.g. (Moghadam and Mozayani, 2011) and (Lai and Qin, 2010)).

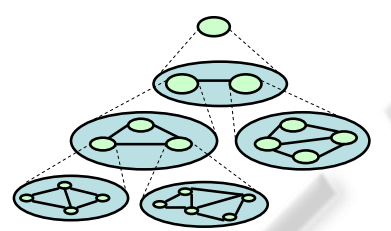

Figure 1: A Holarchy: organization of holons.

\section{THE ENVISIONED SMART GRID}

We model the smart grid as a system formed from interconnection of prosumers of different types and complexities. A prosumer could be as simple as a household that autonomously manages its resources (appliances, distributed sources, and/or storage systems). Other systems such as VPP, microgrid, $\mathrm{AN}$, etc. can also be regarded as prosumers. A prosumer can have a sub-part that is also a prosumer in itself. Our proposed architecture reflects a paradigm shift from a centralized top-down system to a decentralized bottom-up one where prosumers recursively join in different aggregation layers to form the whole power system. The new scenario entails self-similarity because the prosumers exhibit the autonomy to produce, consume, import, and/or export power, irrespective of their aggregation level. The autonomy, aggregation into layers, recursiveness (self-similarity), and dynamic adaptation of a holonic system make it well suited to model the envisioned smart grid. We model each prosumer as a holon and the entire power system as a holarchy. The new power system holarchy has the following major features.

\subsection{Autonomy/Self-Management}

The prosumer holons autonomously manage their own resources and optimize their utilization. Further, a prosumer holon can choose to be part of a bigger prosumer holon and exchange power with its surrounding, or operate as a self-supplying islanded unit. The autonomy of the prosumer holons aids a distributed control capability of the system that would be otherwise very difficult to control centrally. Autonomy might also increase the consciousness of the prosumer holon, making it more cautious about its consumption, which could increase energy efficiency.

\subsection{Bottom-up Organization}

The prosumer holons (such as households) at a lower aggregation level may be self-organized to constitute a bigger prosumer holon (such as a neighbourhood energy community) to locally exchange power with each other and to gain a collective bargaining power with the rest of the grid. For the same reasons, the newly formed prosumer holon can still be connected to its peers in a network in the next higher aggregation layer to form yet a bigger prosumer holon. Further bottom-up grouping of such holons in networks-of-networks continues recursively in higher aggregation levels and eventually constitutes the overall holarchy of the smart grid as shown in Figure 2. Simon (Simon, 1962) has shown that this recursive clustering at different aggregation layers is inherent behaviour of all complex systems. The smaller holons that form a bigger holon are referred to as sub-holons of the bigger holon on the next aggregation layer, whereas, the bigger holon on the next aggregation layer that contains a holon is referred to as its super-holon. The bottom-up organization at different aggregation layers provides efficient structure that abstracts and simplifies the coordination of the system. Moreover, the aggregation of prosumer holons into superholons might increase the reliability of the collective as the profiles of the individual holons might complement each other. Further, the local power 
exchanges between the prosumer holons reduces both the transportation losses and the costly investment costs to upgrade the transmission capacity to meet the ever increasing demands.

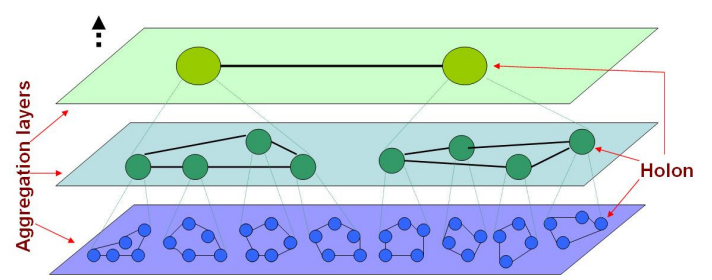

Figure 2: Bottom-up organization of prosumer holons to form the power holarchy.

\subsection{Dynamic Reconfiguration}

Over an interval of time, a prosumer holon may be in the state of either self sufficiency, surplus production, or excess demands. Thus, the prosumer holons in our envisioned system can leave one super-holon to join another one over a period of time to look for a set of holons that complement their profiles, thereby optimizing their resource utilization. It has been shown in (Erol-kantarci et al., 2011) that a set of microgrids could be dynamically reorganized during a day into clusters according to their profiles to optimize the utilization of their renewable resources. Moreover, prosumer holons can join a super-holon for some time intervals during a day and operate in island mode at other intervals, for example in response to potential faults. Such flexibilities lead to a dynamic reconfiguration of the smart grid holarchy over a period of time.

\subsection{Intelligence and Communication}

A prosumer holon needs to coordinate its components, as well as coordinate itself with its surrounding to optimize utilization of resources and to ensure system stability. This coordination involves large information flows and require intelligent information processing units that constitute the "nerve system" of the "organic" power system. Accordingly, each prosumer holon requires communication capabilities and an intelligent device that makes decisions based on the gathered information.

\section{CONTROL ARCHITECTURE OF THE ENVISIONED SMART GRID}

Given the nature of the envisioned power system, its control architecture needs to exhibit the following properties: 1) sufficient autonomy of each prosumer holon to manage its own resources; 2) recursive structure where a prosumer is composed of smaller prosumers at the lower aggregation layer, and at the same time be part of a larger prosumer holon at a higher aggregation layer; 3) layered structure to facilitate coordination between different aggregation layers; 4) dynamic reconfiguration to runtime topology change. The holonic architecture, as explained before, nicely fits the envisioned system because it combines distributed control and a layered coordination structure. It also provides dynamic reconfiguration and recursive structure that best model the system.

\subsection{Holonic Control Architecture}

Our holonic control architecture maintains the proposed structure in the envisioned power system. In the control domain, a control-holon represents the control system of a prosumer holon. Thus, holonic control units will be recursively organized in a bottom-up structure to form a complete controlholarchy of the smart grid. When the prosumer holon has prosumer sub-holons, its control-holon is also composed of the corresponding control-subholons (i.e. control systems of sub-holons). The control-sub-holons can interact with each other, as well as with their control-holon.

Figure 3 depicts the holonic control architecture. As shown in the figure, the control-holon could represent its members in the next aggregation layer as a member of a control-super-holon. The control mechanism involves mutual negotiations between the control-holon and the control-sub-holons. As part of the negotiations, a control-sub-holon might agree to submit part of its autonomy to the controlholon. Once agreements are reached, the controlholon is responsible to enforce the terms and conditions. The power transactions and provision of system services are based on mutual negotiations, and the control-holon can play a coordinating role to maintain the desired overall state of the system. Coordination could be achieved in various ways such as by providing incentives or through local electronic markets within the holon. 


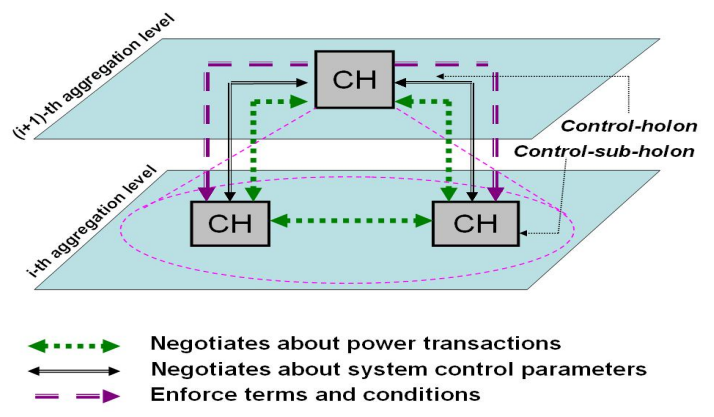

Figure 3: Control architecture of a prosumer holon.

While the prosumer holons are organized in a holarchy as in Figure 2, the control-holon investigates the situations in the external environment and presents the opportunities and threats to its control-sub-holons, and corresponding decisions are made through negotiations. The control-sub-holons also need to translate the negotiations to their corresponding control-subholons (if any), and the process continues recursively in the holarchy. In this manner, the control-holons at various aggregation layers will be conscious about the dynamics in the system and make corresponding optimal decisions through negotiations.

In the holonic control architecture, the aggregation level of a control-holon does not indicate its level of influence. The level of influence of control-holons are decided through contracts that involve negotiations. Accordingly, up on joining a super-holon a control-sub-holon might choose to retain its full autonomy, or submit its autonomy to the control-holon of its super-holon partly or fully.

\subsection{Service Framework of the Holonic Control}

The proposed control paradigm involves a large number of autonomous actors that interact with each other and exchange power. Such a scenario poses various challenges relater to interoperability, scalability, discovery, real-time decision making and others that are best addressed by the Service Oriented Architecture (SOA) (Pagani and Aiello, 2011). SOA is an architecture that models an application as a set of loosely coupled, flexible, reusable and adaptable functional units called services, whereby the services are interrelated through well-defined interfaces and contracts. The main advantages of SOA include platformindependence of the interfaces that aids interoperability, the flexibility and reusability of the services, and its easy adaptation to evolutionary changes in the application. While SOA can be implemented using different technologies, the web services is the most popular one because of the wide acceptance of its standards. Web service technology exposes web services, which are sets of operations, on the web; thereby allowing high degree of integration among them.

Our proposed service framework of the holonic control is composed of four major services.

1. Database service - srvDatabase: This web service takes care of storing, accessing, updating and maintaining the relevant data of the prosumer holon. The data could include topology information, parameter specifications of the grid and the components, history of production, demand and storage profiles, etc.

2. State Evaluation Service srvStateEvaluation: This web service takes care of getting information about the realtime state of the holon and its environment, forecasting the profiles over the next interval(s) of time, and setting appropriate goals depending on the current state and the forecast.

3. Optimization Service - srvOptimization: This service ensures optimal utilization of resources. This could involve devising a coordination mechanism to be proposed to sub-holons to achieve a desirable profile, and finding optimal scheduling of the resources, such as power sources, storage systems, and appliances.

4. Transaction Service - srvTransaction: This service contains operations that support power transactions between prosumer holons. For instance, a prosumer holon that wants to import power can publish its demand, while it can notify others about its interest to export its surplus production. Through such operations the prosumer holons can negotiate and exchange electric power with each other.

5. Stability Service - srvStability: This service is a set of operations that guard the stability of the prosumer holon. It must make sure that the active and reactive power are balanced, the power quality is maintained, and faults are safely recovered.

\section{CONCLUSIONS AND DISCUSSIONS}

The electricity power system is facing an era of 
prosumerization, whereby any stakeholder can autonomously produce, consume, import and/or export power. Since the classical centralized and top-down organized power system cannot efficiently and sustainably handle the new scenario, a new optimal way of structuring the system is essential. We have presented an optimal alternative architecture using the concepts of holons and holarchy. Our architecture organizes the system bottom-up from autonomous prosumers that are recursively clustered at various aggregation layers allowing dynamic reconfiguration. The proposed architecture is generic allowing it to accommodate the heterogeneous composition of the power grid. A corresponding control architecture is also presented together with a service oriented architecture (SOA) based framework that models the holonic control architecture. Currently, we are working on formulating the details of the proposed holonic control architecture, aiming at defining the relevant control functions and designing the appropriate protocols for their interaction with each other as well as with their environment.

The proposed architecture is in line with the trends in prosumerization of the power system. Moreover, it is generic and is capable of accommodating the potentially heterogeneous types of networks in the smart grid. Its implementation poses several requirements though. The physical infrastructures need to be enhanced to accommodate the required level of flexibility. Intelligent solutions are required to enable bidirectional power flows, to easily connect and island prosumers, and to maintain system stability under these circumstances. Further, the prosumer holons need intelligent units that can exchange information across communication infrastructures, process information and make decisions.

Further, modelling the behaviours of the autonomous prosumers is essential to propose coordination mechanisms for optimal system-wide behaviour. On the other hand, an in-depth investigation of the relevant legal issues is essential to design relevant regulations that accommodate the envisioned system.

\section{REFERENCES}

European Parliament (2009). "All New Buildings to be Zero Energy from 2019," Committee on Industry, Research and Energy, Brussels 2009.

Dielmann, K. and van der Velden, A. (2003). Virtual power plants (vpp)-a new perspective for energy generation? In MIT 2003. IEEE.

Erol-Kantarci, M., Kantarci, B., and Mouftah, H. (2011). Reliable overlay topology design for the smart microgrid network. Network, IEEE, 25(5):38 - 43.

Grijalva, S. and Tariq, M. (2011). Prosumer-based smart grid architecture enables a flat, sustainable electricity industry. In ISGT, 2011 IEEE PES. IEEE.

Hatziargyriou, N., Jenkins, N., Strbac, G., Lopes, J., Ruela, J., Engler, A., Oyarzabal, J., Kariniotakis, G., and Amorim, A. (2006). Microgridslarge scale integration of micro-generation to low voltage grids. Session CIGRE.

Huang, A., Crow, M., Heydt, G., Zheng, J., and Dale, S. (2011). The future renewable electric energy delivery and management (freedm) system: The energy internet. Proceedings of the IEEE, 99(1):133-148.

Koestler, A. (1990). The Ghost in the Machine. Penguin Group.

Kok, J., Warmer, C., and Kamphuis, I. (2005). Powermatcher: multiagent control in the electricity infrastructure. In Proc. of the fourth int. joint conf. on Autonomous agents and multiagent systems, pages $75-82$.

Lai, L. and Qin, J. (2010). A method of the manufacturing information integration management based on holonic. In ICSPS, 2010, volume 2, pages V2-123. IEEE.

Moghadam, M. and Mozayani, N. (2011). A street lighting control system based on holonic structures and traffic system. In ICCRD, 2011, volume 1, pages 92-96. IEEE.

Nara, K. and Hasegawa, J. (1997). A new flexible, reliable, and intelligent electrical energy delivery system. Electrical engineering in Japan, 121(1):2634.

Pagani, G. and Aiello, M. (2011). Towards a serviceoriented energy market: Current state and trend. Service-Oriented Computing, pages 203-209.

Provoost, F., Myrzik, J., and Kling, W. (2004). Setting up autonomous controlled networks. In UPEC 2004., volume 3, pages 1190-1194. IEEE.

Simon, H. (1962). Architecture of complex systems. Proceedings of the American Philosophical Society, 106:467-482.

Tsoukalas, L. and Gao, R. (2008). From smart grids to an energy internet: Assumptions, architectures and requirements. In DRPT 2008., pages 94-98. IEEE.

van Overbeeke, F. and Roberts, V. (2002). Active networks as facilitators for embedded generation. Cogeneration and On-Site Power Production, 3(2):37-42. 